\title{
Klotho: a tumor suppressor and modulator of the Wnt/ $\beta$-catenin pathway in human hepatocellular carcinoma
}

\author{
Xiaowei Tang ${ }^{1,2,4}$, Yun Wang ${ }^{1,4}$, Zhining Fan ${ }^{1}$, Guozhong Ji ${ }^{1}$, Min Wang ${ }^{1}$, Jie Lin ${ }^{1}$, Shu Huang ${ }^{1}$ and Stephen J Meltzer ${ }^{3}$
}

Klotho, an anti-aging gene, has recently been shown to contribute to human hepatic tumorigenesis. In addition, it is known that Wnt signaling is antagonized by the protein klotho. Because augmented Wnt signaling has an important role in tumorigenesis of human hepatocellular carcinoma (HCC), we studied the relationship of klotho expression and activity to the Wnt pathway in this malignancy. Immunohistochemical analysis performed on tissue arrays revealed that klotho expression levels were significantly lower in HCC than in adjacent noncancerous tissues, while klotho staining was inversely correlated with clinical stage and histologic grade. Patients with klotho-expressing tumors had longer survival periods than did those with klotho-negative tumors. Overexpression of klotho as well as treatment with soluble klotho protein reduced hepatoma cell growth in vitro and in vivo, whereas klotho silencing enhanced cellular proliferation. Moreover, forced expression of klotho inhibited Wnt/ $\beta$-catenin signaling, as confirmed by reduced expression of $\beta$-catenin, inhibition of translocation of $\beta$-catenin from the cytoplasm to the nucleus, and reduced expression of c-myc and cyclin $D 1$, two known target genes of the Wnt/ $\beta$ catenin pathway. In contrast, activation of the Wnt/ $\beta$-catenin pathway was enhanced when klotho was silenced by inhibitory RNAs. Furthermore, serum levels of soluble klotho in patients with malignant tumors were studied, and results suggested a significant increase in these levels in HCC patients. These data suggest that klotho acts as a tumor suppressor and an inhibitor of the Wnt/ $\beta$-catenin pathway in HCC, and moreover, that soluble klotho is a potential serum biomarker for HCC.

Laboratory Investigation (2016) 96, 197-205; doi:10.1038/labinvest.2015.86; published online 3 August 2015

Primary liver cancer, which consists predominantly of hepatocellular carcinoma (HCC), is the fifth most common cancer worldwide and the third most common cause of cancer mortality. ${ }^{1,2}$ Most HCC cases (>80\%) occur in either subSaharan Africa or in Eastern Asia. China alone accounts for more than $50 \%$ of the world's cases. ${ }^{1}$ The incidence of HCC is also increasing in the western world, including the United States and other countries. ${ }^{3}$ Because HCC is highly aggressive and resistant to chemotherapy and radiotherapy, ${ }^{4}$ surgical resection and liver transplantation are current best curative options to treat liver cancer. However, recurrence or metastasis is quite common in patients who have had a resection and survival rate is $30-40 \%$ at 5 years postoperatively. ${ }^{5,6}$ Thus, in order to develop improved specific or targeted therapies, it is mandatory to understand molecular mechanism underlying hepatocarcinogenesis.
Currently, analyses of genetic alterations have led to the identification of several major signaling pathways that are dysregulated in HCC, including the p53, Rb, and Wnt/ $\beta$-catenin pathways and, to a lesser extent, the TGF- $\beta$ and Met/HGF pathways. ${ }^{6,7}$ Recent studies have also established that aberrations in the $\mathrm{Wnt} / \beta$-catenin signaling pathway are the dominant event in HCC formation. Approximately $20-90 \%$ of HCCs display Wnt/ $\beta$-catenin pathway activation caused by diverse mechanisms. ${ }^{8,9} \beta$-catenin is the chief downstream effector of the canonical Wnt signaling pathway. In the absence of Wnt signal, $\beta$-catenin is destabilized by a multiprotein complex, and then degraded by the proteasome. ${ }^{10}$ Activation of Wnt signaling leads to the accumulation of cytoplasmic $\beta$-catenin, which in turn translocates into the nucleus and interacts with transcription factors of the T-cell factor family to activate target genes,

\footnotetext{
Medical Center for Digestive Diseases, The Second Affiliated Hospital, Nanjing Medical University, Nanjing, China; ${ }^{2}$ Department of Gastroenterology, Nanfang Hospital, Southern Medical University, Guangzhou, China and ${ }^{3}$ Departments of Medicine (Gastroenterology Division) and Oncology, Johns Hopkins University School of Medicine and Sidney Kimmel Comprehensive Cancer Center, Baltimore, MD, USA

Correspondence: Dr S Huang, MD, PhD, Biotherapy Center and Medical Center for Digestive Diseases, The Second Affiliated Hospital, Nanjing Medical University, 121 Jiangjiayuan Road, Nanjing 210011, China or Dr SJ Meltzer MD, Departments of Medicine (Gastroenterology Division) and Oncology, Johns Hopkins University School of Medicine and Sidney Kimmel Comprehensive Cancer Center, 1503 E. Jefferson Street Room\#112, Baltimore, MD 21231, USA.

E-mail: huangshunanjing@hotmail.com or smeltzer@jhmi.edu

${ }^{4}$ These authors contributed equally to this work.

Received 4 September 2014; revised 15 May 2015; accepted 19 May 2015
} 
including $c$-myc and cyclin D1, resulting in abnormal hepatocyte proliferation. ${ }^{11}$

Klotho was identified in 1997 as an anti-aging gene, which can extend life span when overexpressed and induce a premature aging syndrome when disrupted. ${ }^{12,13}$ The klotho gene, whose product is abundantly expressed in various tissues, encodes a single-pass transmembrane protein composed of a large extracellular domain, a transmembrane domain, and a very short intracellular domain. The entire extracellular domain is released into the extracellular space and becomes detectable in blood, urine, and cerebrospinal fluid. ${ }^{14-16}$ Recently, some studies have demonstrated that klotho is related not only to the aging process, but also to human tumorigenesis. For example, klotho functions as a tumor suppressor in breast and pancreatic cancer by inhibiting the insulin and insulin-like growth factor-1 (IGF-1) and fibroblast growth factor (FGF) signaling pathways. ${ }^{17,18}$ Moreover, the extracellular domain of klotho binds to multiple Wnt ligands and inhibits their ability to activate Wnt signaling. ${ }^{12,19}$ The fact that klotho inhibits Wnt signaling may explain several aging-like phenotypes observed in klothodeficient mice. ${ }^{12}$ Similarly, in cervical carcinoma, epigenetic silencing of klotho occurs during the late phase of cervical tumorigenesis, and consequent functional loss of klotho contributes to aberrant activation of the canonical Wnt pathway. ${ }^{20}$ Therefore, we hypothesize that klotho inhibits HCC growth through downregulation of the $\mathrm{Wnt} / \beta$-catenin signaling pathway.

Here, we investigated klotho expression in HCC tissues and sera and its involvement in hepatoma cell proliferation and apoptosis, as well as its activation of the $\mathrm{Wnt} / \beta$-catenin pathway in vitro and in vivo. Our study provides evidence that klotho acts a tumor suppressor and a modulator of the Wnt/ $\beta$-catenin pathway in human HCC.

\section{MATERIALS AND METHODS}

\section{Chemicals, Antibodies, and Constructs}

Recombinant soluble human klotho protein (sKL) was obtained from PeproTech (Rocky Hill, NJ, USA). Antibodies used were $\beta$-catenin, cyclin D1, c-myc, histone $\mathrm{H} 3, \beta$-actin (Epitomics, USA), and klotho (Abcam, USA). Small interfering RNA oligos for knocking down klotho (siRNA- $k l$ ) and non-target control siRNA (siRNAc) were purchased from Santa Cruz Biotechnology. An expression plasmid encoding klotho (pCMV6-KL) and the control vector pCMV6-AC-GFP (pCMV6) were purchased from OriGene Technologies (Rockville, MD, USA). All plasmid constructs were confirmed by DNA sequencing.

\section{Tissue Microarrays and Immunohistochemistry}

HCC tissue microarrays were purchased from Shanghai Outdo Biotechnology (Shanghai, China). These arrays contained 52 cancerous and 54 adjacent noncancerous specimens. Clinical parameters and follow-up information were available on all 52 cases. The expression of klotho in tissues was evaluated by immunohistochemical staining with a klothospecific antibody. Staining was scored according to staining intensity and the percentage of cells stained. The intensity was graded from 0 to III: Grade 0 for negative staining intensity, Grade I for weak staining intensity, Grade II for moderate staining intensity, and Grade III for strong staining intensity. The percentage of klotho-positive cells was graded from 0 to 4 (0, less than $5 \%$ of positive cells; $1,5-25 \% ; 2,26-50 \%$; 3 , $51-75 \% ; 4,76-100 \%)$. Final composite staining scores were calculated as the product of staining intensity multiplied by the percentage of stained cells. Klotho expression was considered positive when this composite score was $>4$. Cases with scores equal to or $<4$ were considered negative.

\section{Cells and Transfections}

The immortalized noncancerous liver cell line L02 and HCC cell lines HepG1, HepG2, and SMMC-7721 were cultured in DMEM supplemented with $10 \%$ FBS in a $5 \% \mathrm{CO}_{2}$ incubator at $37^{\circ} \mathrm{C}$. All transfection was performed using LipofectAMINE 2000 according to the manufacturer's protocol (Invitrogen, Grand Island, NY, USA).

\section{MTT Viability Assay}

$4 \times 10^{3}$ cells/well were plated in 96-well plates and cultured in appropriate culture media. At indicated times, cells were cultured for 2 hours with $500 \mathrm{mg} / \mathrm{ml} \mathrm{3-(4,5-dimethylthiazol-}$ 2-yl)-2,5-diphenyltetrazolium bromide (MTT) reagent (Sigma, St Louis, MO, USA). Medium was aspirated, and cells were dissolved with dimethyl sulfoxide. Absorbance of formazan product was measured by an ELISA reader.

\section{Flow Cytometry}

Cells were harvested from 6-well plates and gently disaggregated to a single-cell suspension. Staining was performed according to the manufacturer's protocol. The rate of cellular apoptosis was analyzed by flow cytometry using an annexin V-FITC/PI kit (BD Biosciences, Franklin Lakes, NJ, USA) following the manufacturer's instructions.

\section{Western Blotting}

Protein extraction from cultured cells and western blot analysis were carried out as previously described. ${ }^{21}$ Western blotting of cytosolic and nuclear fractions of hepatoma cells was notable for specific preparation of nuclear and cytosolic fractions: cells were used for fraction collection via mechanical homogenization. Isolation of nuclei and cytosol was carried out using nuclear and cytoplasmic protein extraction kits according to the manufacturer's instructions (Beyotime, Haimen, China). Primary antibodies used in western blotting included antibodies against klotho, $\beta$-catenin, cyclin D1, c-myc, histone $\mathrm{H} 3$, and $\beta$-actin.

\section{Immunofluorescence Staining}

After transfection with empty plasmid or klotho overexpression vector for $48 \mathrm{~h}$, HepG2 cells were grown on coverslips, 
a
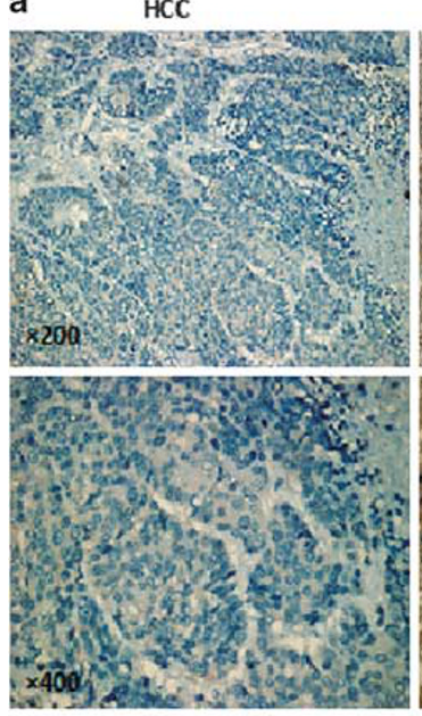

Adjacent normal liver tissue
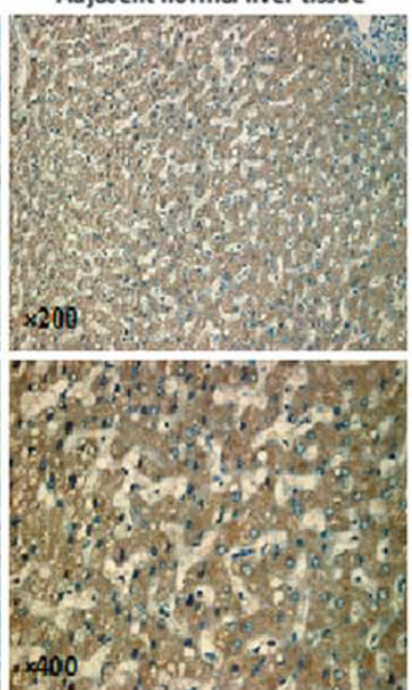

b

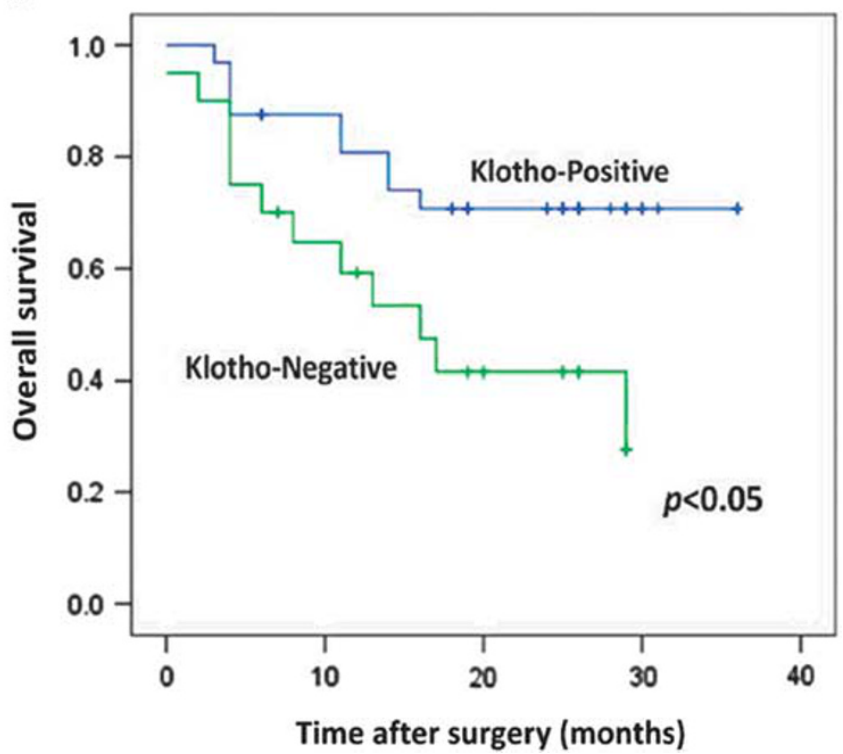

Figure 1 Reduced klotho expression in HCC tissues is associated with reduced survival of HCC patients. (a) Immunohistochemical staining reveals lowklotho expression in HCC tissues (left panels, $\times 200$ and $\times 400$ magnification) and high-klotho expression in adjacent non-tumor liver parenchyma (right panels, $\times 200$ and $\times 400$ magnification). (b) Kaplan-Meier survival analyses show worse survival in HCC patients with klotho-negative ( $n=20)$ than those with klotho-positive tumors $(n=32) ; P<0.05$ by log-rank test. HCC, hepatocellular carcinoma.

rinsed in PBS, and fixed with $4 \%$ formaldehyde in PBS for $20 \mathrm{~min}$ at $4^{\circ} \mathrm{C}$. Cells were permeabilized with PBS containing $0.01 \%$ Triton X-100 and $0.05 \%$ SDS, pH 7.4 for 5 min at $4{ }^{\circ} \mathrm{C}$. Permeabilized cells were rinsed in PBS supplemented with $0.5 \%$ Tween 20 (PBST), and cells were blocked with blocking solution $(0.2 \%$ BSA in PBS, pH 7.4) for $1 \mathrm{~h}$. Cells were rinsed in PBST three times and incubated with either a mixture of anti- $\beta$-catenin mouse monoclonal antibody (1:300 dilution in PBST) and anti-klotho rabbit polyclonal antibody (1:300 dilution in PBST) at $4{ }^{\circ} \mathrm{C}$ overnight. After rinsing in PBST, coverslips were incubated in a mixture of Texas red-conjugated anti-rabbit IgG secondary antibody and fluorescein isocyanate (FITC)-conjugated anti-mouse $\operatorname{IgG}$ secondary antibody (KPL, Gaithersburg, MD, USA), at a 1:200 dilution in PBST for $1 \mathrm{~h}$ at $37^{\circ} \mathrm{C}$. Cell nuclei were counterstained with $10 \mu \mathrm{m} \quad$ 4',6'-diamidino-2-phenylindole (DAPI). The intracellular distributions of the target proteins were analyzed using confocal fluorescent microscopy. Confocal images were sequentially acquired with Zeiss AIM software on a Zeiss LSM 510 confocal microscope system (Carl Zeiss, Thornwood, NY, USA) with excitation at $488 \mathrm{~nm}$ (for FITC), $543 \mathrm{~nm}$ (for Texas red), and $340 \mathrm{~nm}$ (for DAPI). Negative controls without primary antibody were also prepared.

\section{Serum Samples}

A total of 20 patients with newly diagnosed HCC, referred to the Second Affiliated Hospital of Nanjing Medical University from December 2011 to December 2012, were considered eligible for this study. To exclude the possible confounding influences of age, sex, and aging-associated basic diseases, the patient characteristics should be matched between HCC group and the controls. Thereupon, 29 subjects who visited the hospital for their annual physical examinations were assigned to a control group. For inclusion criteria, results of their physical examinations had to reveal no evidence of malignancy. Written informed consent was obtained for the acquisition and use of serum samples and clinical data. Ethical approval was granted by the Ethics Committee of the Second Affiliated Hospital of Nanjing Medical University.

\section{ELISA Assays}

Blood samples were collected in the morning after a 12-hour fast. Aliquots of serum were immediately obtained and stored at $-80^{\circ} \mathrm{C}$. Serum soluble klotho levels were measured using an ELISA kit purchased from Immuno-Biological Laboratories (Gunma, Japan). Intra- and inter-assay coefficients of variation ranged from 2.7 to $9.8 \%$. The minimum level of detectability of the assay was $6.15 \mathrm{pg} / \mathrm{ml}$.

\section{Animal Studies}

Mouse maintenance and experiments were carried out under the institutional guidelines of Nanjing Medical University. Athymic nude mice (BALB/c nu/nu, 5 weeks old) were injected with hepatoma cells SMMC-7721 $\left(2 \times 10^{6}\right.$ cells $)$ subcutaneously into one flank. Tumor size was measured with a digital caliper for up to 5 weeks, and volume was estimated using the equation: tumor volume $\left(\mathrm{mm}^{3}\right)=0.5 \times$ length $(\mathrm{mm}) \times$ width $(\mathrm{mm}) \times$ width $(\mathrm{mm})$. When tumors measured an average volume of $80 \mathrm{~mm}^{3}$, mice were treated with intratumoral injections of sKL $7 \mu \mathrm{g} / \mathrm{kg}$ or saline control twice 
weekly for 4 weeks ( $n=5$ per group, three male and two female).

\section{Statistical Analyses}

Experimental results were presented as mean \pm s.e.m. Categorical variables were compared between study groups using Fisher's exact test, while continuous variables were compared using $t$-test. Survival was calculated from date of surgery to date of death or last follow-up. Cumulative survival rate was calculated by the Kaplan-Meier method, and differences in survival were estimated with the log-rank test. Cox proportional hazards modeling was used for multivariate survival analysis. $P$-values $<0.05$ were considered significant. All statistical analyses were performed using SPSS version 18.0 (SPSS, Chicago, IL, USA) software for Windows.

\section{RESULTS}

\section{Reduced Klotho Expression in HCC}

To investigate the clinical significance of klotho expression in hepatocarcinogenesis, immunohistochemical analyses of klotho expression were conducted on HCC tissue arrays, containing 52 tumor specimens and 54 adjacent non-tumor specimens. Positive klotho expression was observed in 53 (98.1\%) of normal liver samples adjacent to HCC, compared with $32(61.5 \%)$ of HCC samples $(P<0.05$, Figure 1a).

An examination of clinical and pathological tumor characteristics of the 52 HCC samples revealed that klotho expression correlated inversely with histological grade and clinical stage $(P<0.05)$, but not with age, gender, tumor size, liver cirrhosis, or invasion depth (Table 1). Moreover, Kaplan-Meier survival analysis indicated that overall survivals of hepatoma patients with positive klotho expression were significantly longer than those with negative klotho expression $(P<0.05$, Figure $1 b)$. Klotho expression in cell lines was also analyzed by western blotting. Klotho expression was higher in the immortalized normal liver cell line L02 than in HCC cell lines HepG1, HepG2, and SMMC-7721 (Figures 2a and $\mathrm{b}$ ).

\section{Klotho Overexpression Inhibits, Whereas Klotho Downregulation Enhances, Hepatoma Cell Growth}

To explore the potential function of klotho in the growth of hepatoma cells, a series of cell culture models should be established. Two common kinds of HCC cell lines, HepG2 and SMMC-7721, express low levels of klotho protein. However, western blot revealed very-low klotho expression in SMMC-7721 cells in comparison with HepG2 (Figure 2b), so silencing of klotho in SMMC-7721 cells was not feasible. Moreover, as HepG2 cells are more susceptible to HBV (hepatitis $\mathrm{B}$ virus) infection, while $\mathrm{HBV}$ infection is a primary cause of HCC in Chinese people, the results from HepG2 cells may better match the findings of tissue microarrays of HCC patients. Therefore, we chose HepG2 cells as our research object in vitro.
Table 1 Associations of klotho expression with clinicopathologic variables

\begin{tabular}{lcccc}
\hline & & \multicolumn{2}{c}{ Klotho expression } & \\
\cline { 3 - 3 } Group & Number & Positive $(n, \%)$ & Negative $(n)$ & $P$-value \\
\hline Age (years) & & & & \\
$\geq 60$ & 10 & $5(50)$ & 5 & 0.404 \\
$<60$ & 42 & $27(64.3)$ & 15 & \\
Gender & & & & \\
Female & & & & \\
Male & 6 & $3(50)$ & 3 & 0.537 \\
& 46 & $29(63.0)$ & 17 &
\end{tabular}

$\begin{array}{ccccc}\begin{array}{l}\text { Tumor size }(\mathrm{cm}) \\ \leq 5\end{array} & 24 & 17(70.8) & 7 & 0.202 \\ >5 & 28 & 15(53.6) & 13 & \end{array}$

$\begin{array}{lcccc}\text { Histological grade } & & & \\ \text { Well (I, I-II) } & 8 & 8(100) & 0 & 0.002^{\mathrm{a}} \\ \text { Moderately (III, II-III) } & 34 & 22(64.7) & 12 & \\ \text { Poorly (III) } & 8 & 2(25.0) & 6 & \\ \text { Clinical stage } & & & & \\ \text { 1, } 2 & 44 & 39(70.5) & 13 & 0.007^{\mathrm{a}} \\ 3,4 & 8 & 1(12.5) & 7 & \\ \text { Invasion depth } & & & & \\ \text { T1, T2 } & & & & \\ \text { T3 } & 46 & 31(67.4) & 15 & 0.05 \\ & 6 & 1(16.7) & 5 & \\ \text { Liver cirrhosis } & & & & \\ \text { No } & & 16(64.0) & 9 & 0.726 \\ \text { Yes } & 27 & 16(59.3) & 11 & \end{array}$

${ }^{\mathrm{a}} P<0.05$ was considered statistically significant.

Firstly, HepG2 cells were transfected with either a klotho expression vector (pCMV6-KL) or an empty vector (pCMV6) for $48 \mathrm{~h}$. Transfection efficiency was assayed at $>85 \%$ according to the expression of GFP (data not shown). As shown in Figure 2c, klotho expression in HepG2 cells was markedly increased after transfection with pCMV6-KL. In klotho-expressing cells, proliferation (evaluated by MTT assays) was reduced to $43 \%$ (Figure $2 \mathrm{~d}$ ), whereas apoptosis (as assessed by flow cytometry) was increased 2.3-fold compared with control cells (Figure 2e). 
a

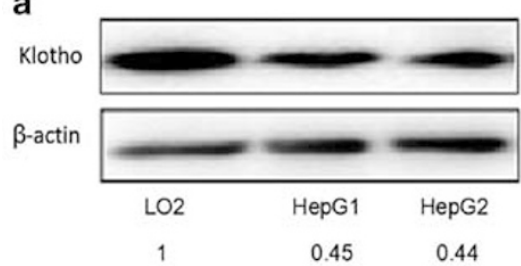

d

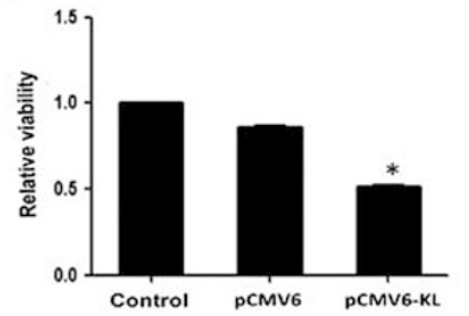

b

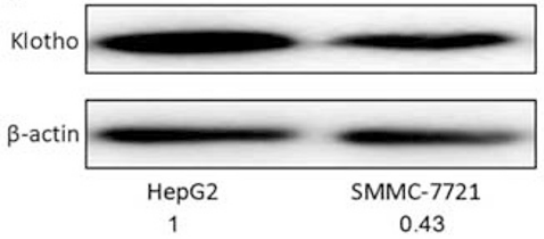

1

0.43

e-1
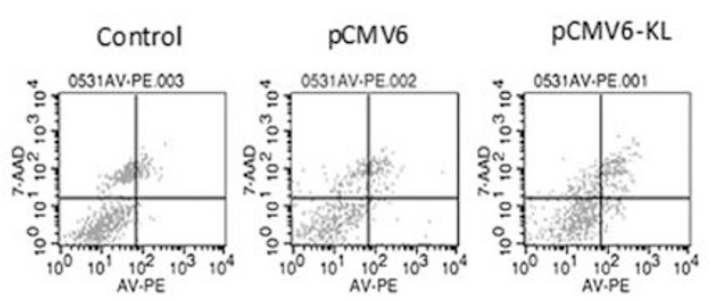

c

Klotho

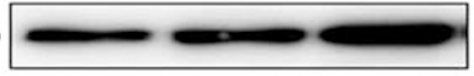

$\beta$-actin

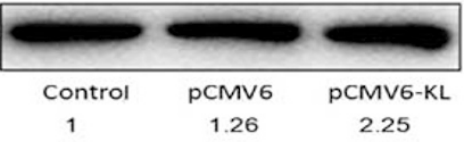

e-2

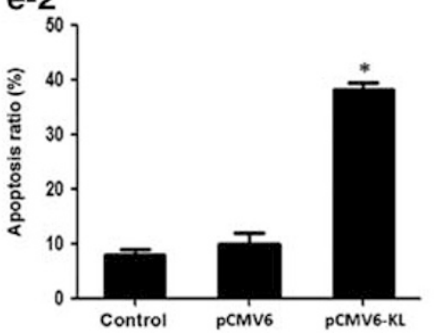

Figure 2 Forced overexpression of klotho inhibits HCC cell growth. (a) Western blotting analysis of native klotho protein expression levels in L02, HepG1, and HepG2 cell lines. (b) Western blotting analysis of klotho protein expression levels in HepG2 and SMMC-7721 cell lines. (c) Klotho protein level measured by western blot, (d) cellular viability by MTT, and (e) apoptosis by flow cytometry, respectively, in HepG2 cells transiently transfected with empty vector (pCMV6) or a klotho overexpression vector (pCMV6-KL) for $48 \mathrm{~h}$. Western blot bands are representative of three independent experiments. The bars represent means of three independent replicates per group, with s.e. bars ( ${ }^{*}$-test $P<0.05$ ). HCC, hepatocellular carcinoma.

a

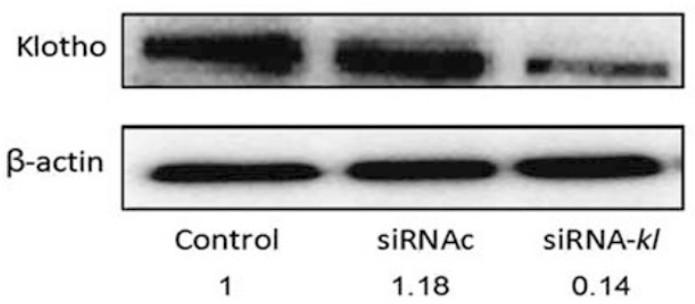

c-1

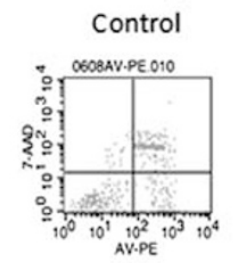

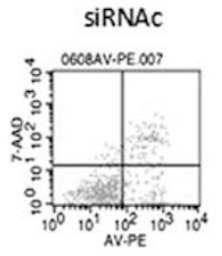

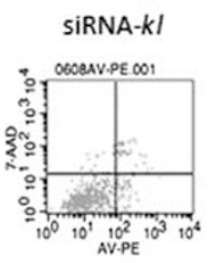

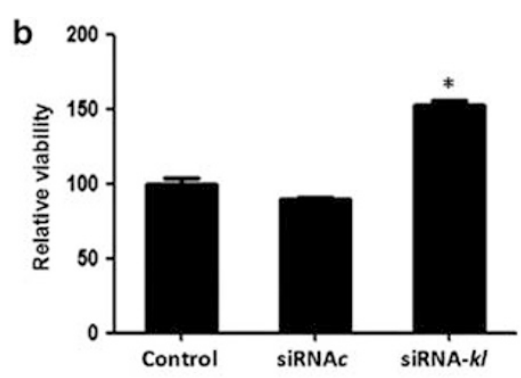

c-2

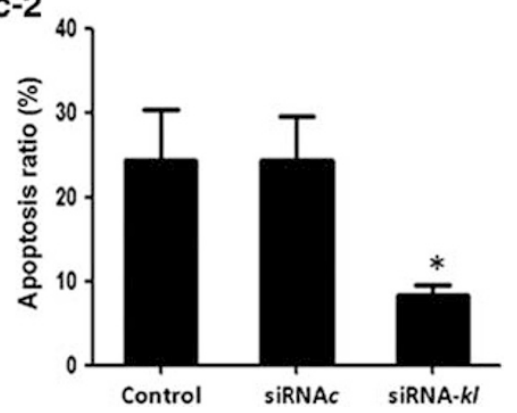

Figure 3 Knockdown of klotho enhances HCC cell growth. Klotho protein expression measured by western blotting (a), cellular viability by MTT (b), and apoptosis by flow cytometry (c), respectively, in HepG2 cells transfected with a nonspecific siRNA (siRNAc) or a klotho-specific siRNA (siRNAc-kl) for $48 \mathrm{~h}$, respectively. Western blotting bands are representative of three independent experiments. Bars represent means of three independent replicates per group, with s.e. bars $\left({ }^{*} t\right.$-test $\left.P<0.05\right)$. HCC, hepatocellular carcinoma.

Because some degree of klotho expression was observed in HepG2 cells (Figure 2a), we next examined the effects of klotho downregulation in these cells. Analyses of klotho protein levels following transfection with a klotho-directed inhibitory RNA (siRNA- $k l$ ) revealed significant reduction of klotho expression, compared with a siRNAc (Figure 3a). Subsequent results showed that knockdown of klotho using
siRNA- $k l$ enhanced the growth of HepG2 cells (Figure 3b), but did not affect the rate of apoptosis (data not shown).

\section{Klotho Inhibits Activation of the Wnt/ $\beta$-Catenin Pathway in Hepatoma Cells}

As the Wnt $/ \beta$-catenin pathway is known to have a key role in the development of HCC, ${ }^{8,9}$ we analyzed the effect of klotho 
a

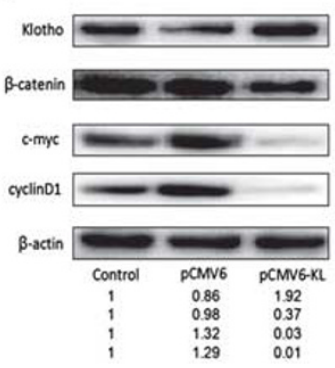

d

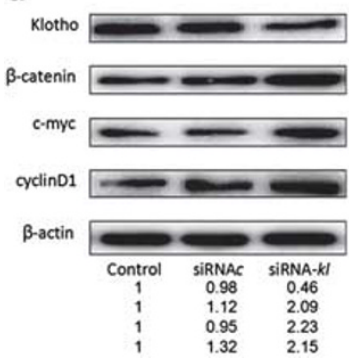

b

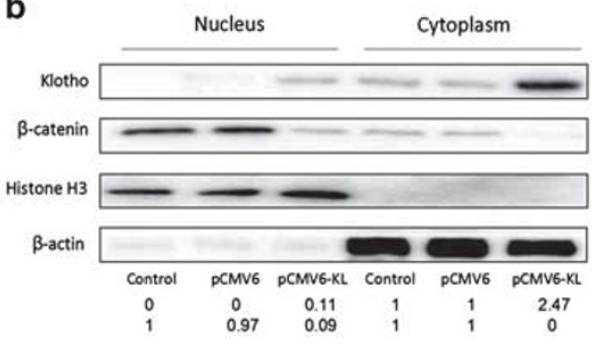

e

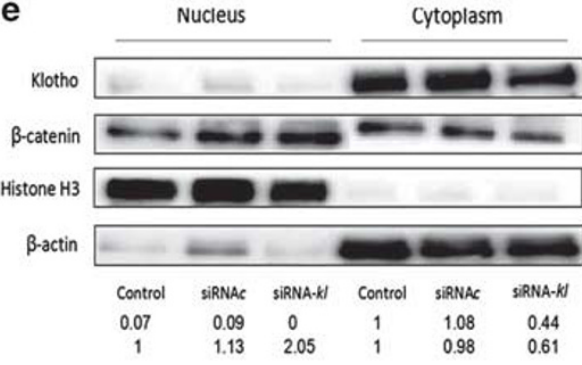

c

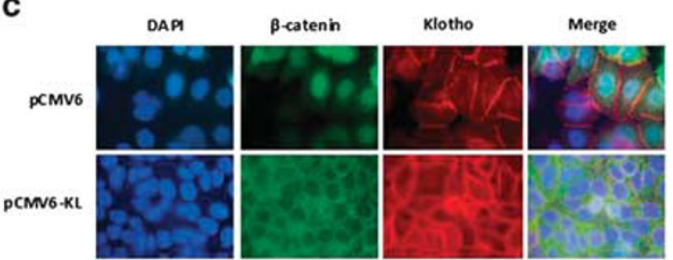

Figure 4 Blockage of Wnt signaling by klotho in HCC cells. Western blots of whole-cell (a and $\mathbf{d}$ ), cytosolic (b and e), and endonuclear (b and e) fractions reveals the expression levels of $\beta$-catenin, c-Myc, and cyclin D1 in HepG2 cells transfected with klotho plasmid (a and b) or klotho-specific siRNA ( $\mathbf{d}$ and $\mathbf{e}$ ) for $48 \mathrm{~h}$, respectively. $\beta$-actin was used as a whole-cell and cytoplasmic internal standard, while histone $\mathrm{H} 3$ was used as a nuclear internal standard to exclude cross-contamination during subcellular fraction isolation. Bands are representative of three independent experiments. (c) Representative immunofluorescence images show inhibition of translocation of $\beta$-catenin from the cytoplasm to the nucleus in HepG 2 cells transfected with klotho plasmid vs empty vector for $48 \mathrm{~h}$, respectively. $\beta$-catenin is labeled green, while nuclei were blue counterstained with DAPI and membranes were red with anti-klotho antibody. Magnification $400 \times$. HCC, hepatocellular carcinoma.

on activation of the $\mathrm{Wnt} / \beta$-catenin pathway in hepatoma cells. HepG2 cells were transfected with either a klotho plasmid or an empty vector for $48 \mathrm{~h}$, then analyzed by western blotting for expression of downstream components of the Wnt pathway. Results of these assays revealed that klotho overexpression reduced levels of the downstream genes $\beta$-catenin (in whole cells, as well as in nuclear fractions), c-myc, and cyclin D (Figures $4 \mathrm{a}$ and b). In particular, immunofluorescence staining was used to visualize subcellular localization of $\beta$-catenin. This experiment revealed that $\beta$-catenin nuclear translocation was inhibited after transfection with klotho $v s$ empty vector (Figure $4 \mathrm{c}$ ). Thus, these data indicate the inhibition of the $\mathrm{Wnt} / \beta$-catenin pathway by klotho overexpression in hepatoma cells.

Conversely, inhibition of klotho using klotho-directed siRNA increased the levels of the Wnt downstream targets $\beta$-catenin (in whole cells, as well as in nuclear fractions), c-myc, and cyclin D, suggesting the enhancement of Wnt signaling pathway activation (Figures $4 \mathrm{~d}$ and e).

\section{sKL Inhibits Hepatoma Cell Growth In Vitro and In Vivo}

Klotho may be secreted from cells, where it may act as a circulating hormone. ${ }^{12}$ We therefore tested the ability of sKL to inhibit hepatoma cell growth. HepG2 cells were treated with commercially available sKL, and cell viability was evaluated by MTT assay. As showed in Figure 5a, after $48 \mathrm{~h}$ exposure of HepG2 cells to sKL at concentrations of 0.25 ,
$0.50,0.75,1.00$, and $1.25 \mu \mathrm{g} / \mathrm{ml}$, cell growth was reduced in a dose-dependent manner $(P<0.05)$. Moreover, downregulation of $\beta$-catenin, $\mathrm{c}-\mathrm{myc}$, and cyclin D1 protein expression in HepG2 cells treated with sKL $(1.00 \mu \mathrm{g} / \mathrm{ml}, 48 \mathrm{~h})$ indicated the inhibition of $\mathrm{Wnt} / \beta$-catenin pathway activation (Figure $5 \mathrm{~b}$ ).

As HepG2 cells were not feasible for establishing HCC in nude mice, whereas SMMC-7721 cells were. Therefore, we applied SMMC-7721 cells in our animal study instead of HepG2 cells. SMMC-7721 cells were injected into one flank of athymic mice to test the growth inhibitory activity of sKL in vivo. Tumor size was measured with a digital caliper and volume was estimated. When tumors measured an average volume of $80 \mathrm{~mm}^{3}$, HCC-bearing mice were treated by intratumoral injections of either control vehicle $(n=5)$ or sKL ( $7 \mu \mathrm{g} / \mathrm{kg}, n=5)$, twice weekly for 4 weeks. Administration of sKL did not affect total animal weight (Figure 5c) but did significantly decrease tumor size and weight (Figures $5 \mathrm{~d}-\mathrm{f}$, $P<0.05$ for comparison between treated and control groups).

\section{Increased Serum Levels of Klotho in HCC Patients}

We employed ELISA assays to measure soluble klotho levels in sera of 20 HCC patients and 29 control subjects. Potential confounding factors for serum klotho levels in subjects and controls are shown in Table 2: differences were not statistically significant. Higher serum klotho levels were noted in HCC patients (mean \pm s.e.m., $291.60 \pm 30.57 \mathrm{pg} / \mathrm{ml}$ ) than in control subjects (mean \pm s.e.m., $186.06 \pm 14.28 \mathrm{pg} / \mathrm{ml}$; 


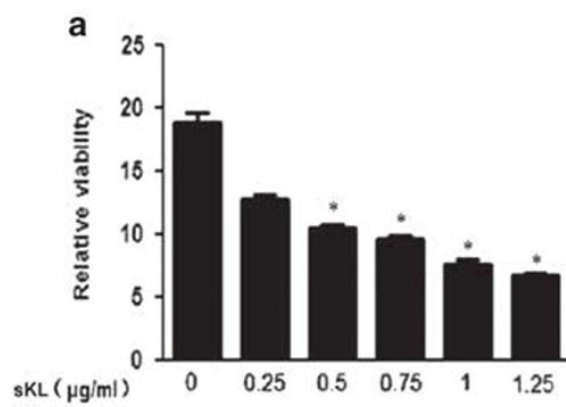

b

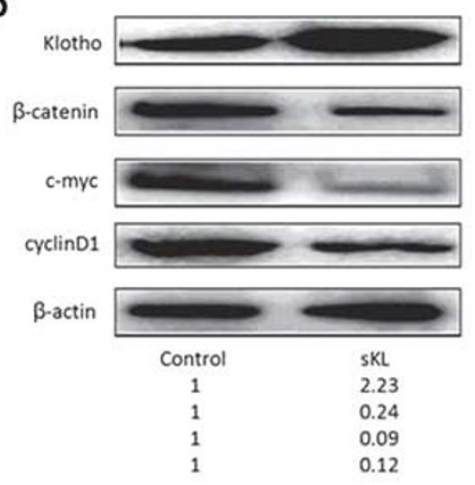

d

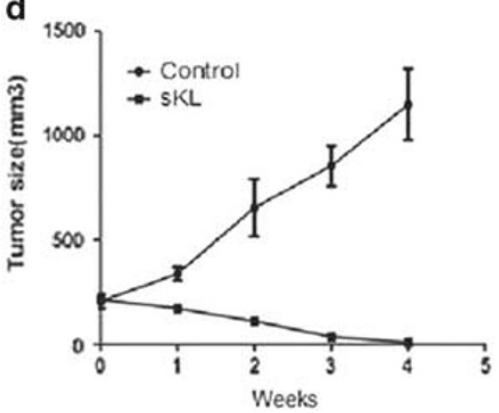

e

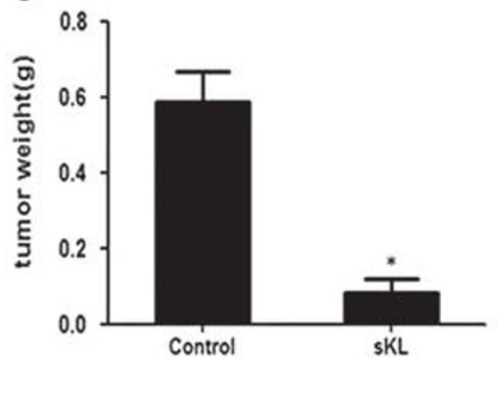

C

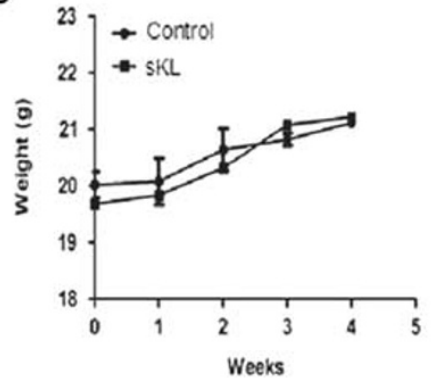

f

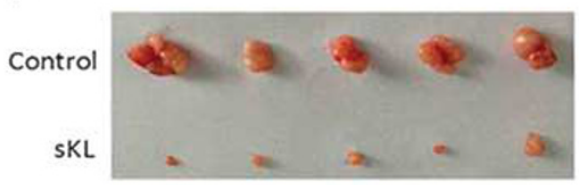

Figure 5 sKL inhibits tumor growth in vitro and in vivo. (a) Diminished viability of HepG2 cells treated with sKL. MTT assays were conducted at $48 \mathrm{~h}$. The bars represent means of three independent replicates per group, with s.e. bars. ${ }^{*} P<0.05$ for treated cells vs control. (b) Diminished levels of $\beta$-catenin, c-Myc, and cyclin D1 protein in HepG2 cells treated with sKL $(1.00 \mu \mathrm{g} / \mathrm{ml})$ for $48 \mathrm{~h}$. Western blotting bands are representative of three independent experiments. (c) Whole-animal weight in HCC-bearing mice treated by intratumoral injections of sKL $(7 \mu \mathrm{g} / \mathrm{kg}$ ) or saline control twice weekly for 4 weeks ( $n=5$ per group). Weight was measured weekly. (d) Diminished tumor volume in sKL-treated vs control mice, monitored weekly. (e) Diminished tumor weight and (f) grossly visible tumor size in sKL-treated vs control mice, measured on the day of sacrifice $(* P<0.05)$.

HCC, hepatocellular carcinoma; sKL, soluble human klotho protein.

Table 2 Characteristics of HCC patients and control subjects examined for klotho serum levels

\begin{tabular}{lccc}
\hline Characteristic & Control group $(n=29)$ & HCC group $(n=20)$ & $P$-value \\
\hline Age (years) & $58.21 \pm 2.08$ & $56.05 \pm 2.54$ & 0.513 \\
Gender (men) & 20 & 13 & 0.771 \\
Hypertension & 11 & 6 & 0.566 \\
Diabetes mellitus & 4 & 2 & 1.000 \\
Kidney disease & 1 & 1 & 0.161
\end{tabular}

$P<0.05$, Figure 6a). To further evaluate the potential utility of serum klotho levels as a diagnostic test for HCC, receiver operating characteristic (ROC) curve analyses were performed, which yielded a cutoff serum klotho value of $227.90 \mathrm{pg} / \mathrm{ml}$ with a sensitivity of $71.4 \%$ and a specificity of $72.4 \%$ in 20 HCC patients vs 29 controls (Figure $6 \mathrm{~b}$ ). The area under the ROC curve was 0.759 , with $P<0.05$. These findings suggest that soluble klotho levels represent a potential diagnostic serum biomarker of HCC.

\section{DISCUSSION}

The klotho gene, named after the Greek goddess Klotho who spun the thread of life, was originally identified as a putative age-suppressing gene. $^{22}$ Subsequently, various functional aspects of the klotho gene have been investigated, leading to the identification of multiple novel endocrine axes that regulate various metabolic processes and an unexpected link between mineral metabolism and aging. ${ }^{22}$ The observations of Wolf et $a l^{17}$ identified, for the first time, tumor-suppressive activity for klotho in breast cancer in 2008. Since that publication, the role of klotho in tumorigenesis, cancer progression, and prognosis has attracted increasing attention. ${ }^{23}$ Recent studies have shown that klotho participates in the progression of several types of human cancer, including lung cancer, gastric cancer and pancreatic adenocarcinoma. ${ }^{23}$ In the majority of these malignant tumors, klotho acts as a tumor suppressor gene. Nevertheless, some controversial results have also been observed. For example, high expression of secreted klotho was associated with an increased risk of disease progression and death from ovarian cancer. $^{24}$

In HCC in particular, contradictory roles of klotho have been reported by three recent studies. Xie et $a l^{25}$ found that epigenetic silencing of klotho expression correlates with a poor prognosis in HCC, suggesting a tumor-suppressive function for klotho. Similarly, Shu et al ${ }^{26}$ reported that restoration of klotho expression induces apoptosis and 

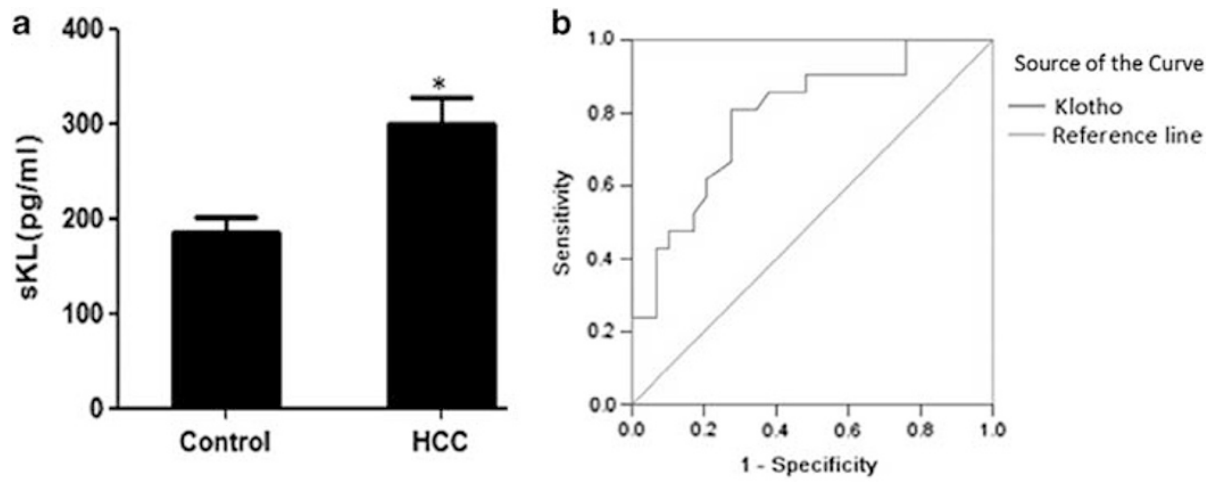

Figure 6 Serum levels of soluble klotho in HCC patients. (a) Klotho serum levels in HCC patients $(n=20)$ were significantly higher than in control subjects $(n=29)$. (b) Receiver operating characteristic (ROC) curve analysis of serum klotho levels in $20 \mathrm{HCC}$ patients vs 29 controls $(* P<0.05)$. HCC, hepatocellular carcinoma.

autophagy in HCC cells. However, the study by Chen et al ${ }^{27}$ revealed a novel oncogenic function of klotho in promoting anoikis resistance via the activation of VEGFR2/PAK1 signaling, thereby facilitating tumor migration and invasion during hepatoma progression. These existing studies suggest that the role of klotho in HCC development may be complex, but that further clarification is needed. In the present study, we studied the expression and activity of klotho in tissues and sera of HCC patients, hepatoma cell lines in vitro, and HCCbearing nude mouse in vivo. Immunohistochemical analyses of tissue arrays revealed reduced expression of klotho in HCC $v s$ adjacent noncancerous tissues. Moreover, staining levels of klotho in HCC were inversely correlated with clinical stage and histologic grade, but positively correlated with overall survival. Similarly, overexpression of klotho reduced hepatoma cell growth and promoted cell apoptosis, whereas klotho silencing enhanced cell proliferation in vitro. Finally, treatment with soluble klotho protein strikingly inhibited hepatoma cell growth both in vitro and in vivo. Therefore, our observations consistently suggest that klotho acts as a tumor suppressor in hepatocarcinogenesis, and moreover that sKL may be useful as a potential strategy for the development of novel therapeutic interventions in HCC patients.

Previous studies have indicated that klotho exerts an anticancer role mainly via inhibition of tumor-related signal transduction pathways, such as the insulin/IGF-1R and FGF cascades. ${ }^{17,18}$ Hepatocarcinogenesis is a multi-step process, with the accumulation of genetic and epigenetic events occurring in a stepwise fashion during tumor progression. ${ }^{6,28}$ In this context, aberrant activation of the $\mathrm{Wnt} / \beta$-catenin signaling pathway has been shown to have a key role in the pathogenesis of HCC. ${ }^{29}$ Secreted klotho protein has been reported to bind to several Wnt ligands and to inhibit Wnt signaling by preventing Wnt from binding to its cognate cellsurface receptor. ${ }^{12,19}$ Klotho also acts as a Wnt antagonist, and klotho gene mutants displayed enhanced Wnt signaling along with stem cell depletion. ${ }^{19}$ In cervical carcinoma, epigenetic silencing of klotho occurs during late phases of cervical tumorigenesis, and consequent functional loss of klotho contributes to aberrant activation of the canonical Wnt pathway. ${ }^{20}$ Therefore, in the present study, we sought to elucidate regulation by klotho of $\mathrm{Wnt} / \beta$-catenin pathway activation in HCC. Our results revealed that forced expression of klotho inhibited the activation of Wnt $/ \beta$-catenin signaling. In contrast, activation of the $\mathrm{Wnt} / \beta$-catenin pathway was enhanced when klotho was silenced by siRNA. These data suggest that klotho acts as an inhibitor of the $\mathrm{Wnt} / \beta$-catenin pathway in HCC.

Klotho exists in both membrane-bound and secreted forms, the latter known as soluble klotho. ${ }^{14-16}$ Secreted klotho can be transcribed through alternative splicing or generated by shedding from the extracellular domain of its transmembrane form. ${ }^{12,30}$ Recently, a sandwich ELISA for the measurement of soluble klotho in humans was established and is now commercially available. ${ }^{31,32}$ Soluble klotho is inversely related to age in healthy subjects and with mortality in the elderly. ${ }^{31,32}$ High levels of soluble klotho occur in human umbilical cord blood. ${ }^{31,33}$ Sze et al ${ }^{34}$ first reported dramatically increased soluble klotho levels in an acquired disease (acromegaly) in humans. In their study, reversal of acromegalic symptoms following tumor removal suggested a causal relationship between the GH-producing adenoma and high-serum klotho levels in acromegaly. ${ }^{34}$ In the present study, to our knowledge for the first time, serum levels of soluble klotho in malignant tumors have been analyzed. To exclude the possible confounding influences of age, sex, and aging-associated basic diseases, these patient characteristics were first matched between HCC group and the controls. Our ELISA results showed that serum klotho levels in HCC patients were significantly higher than in control subjects. Considering the reduced klotho expression levels seen in HCC tissues, mechanisms underlying inconsistent klotho expression patterns observed in HCC tissues and sera remain unclear. It is also poorly understood why cancer development is not impaired by high levels of serum klotho and expression of secreted klotho in normal liver tissue surrounding cancer cells in HCC patients. One possible explanation for this phenomenon is reduced penetrance of soluble klotho to the 
tumor, due to the presence of desmoplastic stroma around hepatoma cells, which is known to confer resistance to cancer therapies. ${ }^{35}$ This hypothesis, to an extent, is supported in the present study by the finding that intratumoral sKL injections significantly decreased tumor size and weight in HCC-bearing nude mice.

In summary, results of the present study support the role of klotho as a tumor suppressor and a modulator of the $\mathrm{Wnt} / \beta$-catenin pathway in HCC. Moreover, expression patterns of klotho in tissues and sera of HCC patients suggest that klotho represents a potential biomarker for the diagnosis of HCC. Finally, in view of the ability of recombinant sKL to inhibit hepatoma cell growth in vitro and in vivo, intratumoral injection or interventional treatment with sKL may, in the near future, constitute novel therapeutic strategies in human HCC patients.

\section{ACKNOWLEDGMENTS}

This work is supported in grants from the National Natural Science Foundation of China (81001076), Natural Science Foundation of Jiangsu Province (BK2012481), Jiangsu Innovation of Medical Team and Leading Talents Cultivation (LJ201127), and USPHS grants CA146799, CA133012, and DK087454.

\section{DISCLOSURE/CONFLICT OF INTEREST}

The authors declare no conflict of interest.

1. El-Serag HB, Rudolph KL. Hepatocellular carcinoma:epidemiology molecular carcinogenesis. Gastroenterology 2007;132:2557-2576.

2. Thomas MB, Jaffe D, Choti $M M$ et al. Hepatocellular carcinoma: consensus recommendations of the National Cancer Institute Clinical Trials Planning Meeting. J Clin Oncol 2010;28:3994-4005.

3. Bosch FX, Ribes J, Dia ZM et al. Primary liver cancer: worldwide incidence and trends. Gastroenterology 2004;127:S5-S16.

4. Tanaka S, Arii S. Molecular targeted therapy for hepatocellular carcinoma in the current and potential next strategies. J Gastroenterol 2011;46:289-296.

5. Blum H. Hepatocellular carcinoma: therapy and prevention. World J Gastroenterol 2005;11:7391-7400.

6. Aravalli RN, Steer CJ, Cressman EN. Molecular mechanisms of hepatocellular carcinoma. Hepatology 2008;48:2047-2063.

7. Laurent-Puig P, Zucman-Rossi J. Genetics of hepatocellular tumors. Oncogene 2006;25:3778-3786.

8. Merle $\mathrm{P}$, de la Monte $\mathrm{S}, \mathrm{Kim} \mathrm{M}$ et al. Functional consequences of frizzled-7 receptor overexpression in human hepatocellular carcinoma. Gastroenterology 2004;127:1110-1122.

9. Ban KC, Singh H, Krishnan R et al. GSK-3beta phosphorylation and alteration of beta-catenin in hepatocellular carcinoma. Cancer Lett 2003;199:201-208.

10. Monga SP. Role of Wnt/ $\beta$-catenin signaling in liver metabolism and cancer. Int J Biochem Cell Biol 2011;43:1021-1029.

11. Thompson MD, Monga SP. WNT/beta-catenin signaling in liver health and disease. Hepatology 2007;45:1298-1305.

12. Wang $Y$, Sun Z. Current understanding of klotho. Ageing Res Rev 2009;8:43-51.

13. Kuro-o M, Matsumura $\mathrm{Y}$, Aizawa $\mathrm{H}$ et al. Mutation of the mouse klotho gene leads to a syndrome resembling ageing. Nature 1997;390:45-51.
14. Chang $Q$, Hoefs $S$, van der Kemp AW et al. The $\beta$-glucuronidase klotho hydrolyzes and activates the TRPV5 channel. Science 2005;310: 490-493.

15. Chen CD, Podvin S, Gillespie $\mathrm{E}$ et al. Insulin stimulates the cleavage and release of the extracellular domain of Klotho by ADAM10 and ADAM17. Proc Natl Acad Sci USA 2007;104: 19796-19801.

16. Imura A, Iwano A, Tohyama $\mathrm{O}$ et al. Secreted Klotho protein in sera and CSF: implication for post-translational cleavage in release of Klotho protein from cell membrane. FEBS Lett 2004;565:143-147.

17. Wolf I, Levanon-Cohen S, Bose $S$ et al. Klotho: a tumor suppressor and a modulator of the IGF-1 and FGF pathways in human breast cancer. Oncogene 2008;27:7094-7105.

18. Abramovitz L, Rubinek T, Ligumsky $\mathrm{H}$ et al. KL1 Internal Repeat Mediates KL Tumor Suppressor Activities and Inhibits bFGF and IGF-I Signaling in Pancreatic Cancer. Clin Cancer Res 2011;17: 4254-4266.

19. Liu H, Fergusson MM, Castilho RM et al. Augmented Wnt signaling in a mammalian model of accelerated aging. Science 2007;317: 803-806.

20. Lee J, Jeong DJ, Kim J et al. The anti-aging gene KLOTHO is a novel target for epigenetic silencing in human cervical carcinoma. Mol Cancer 2010;9:109.

21. Huang $X$, Huang S, Zhang $F$ et al. Lentiviral-mediated Smad4 RNAi promotes SMMC-7721 cell migration by regulation of MMP-2, VEGF and MAPK signaling. Mol Med Report 2010;3:295-299.

22. Kuro-o M. Klotho and the aging process. Korean J Intern Med 2011;26: 113-122.

23. Xie B, Chen J, Liu B et al. Klotho Acts as a Tumor Suppressor in Cancers. Pathol Oncol Res 2013;19:611-617.

24. Lu L, Katsaros D, Wiley A et al. Klotho expression in epithelial ovarian cancer and its association with insulin-like growth factors and disease progression. Cancer Invest 2008;26:185-192.

25. Xie B, Zhou J, Yuan L et al. Epigenetic silencing of Klotho expression correlates with poor prognosis of human hepatocellular carcinoma. Hum Pathol 2013;44:795-801.

26. Shu G, Xie B, Ren F et al. Restoration of klotho expression induces apoptosis and autophagy in hepatocellular carcinoma cells. Cell Oncol (Dordr) 2013;36:121-129.

27. Chen L, Liu H, Liu J et al. Klotho endows hepatoma cells with resistance to anoikis via VEGFR2/PAK1 activation in hepatocellular carcinoma. PLoS One 2013;8:e58413.

28. Kondoh N, Wakatsuki T, Hada A et al. Genetic and epigenetic events in human hepatocarcinogenesis. Int J Oncol 2001;18:1271-1278.

29. Dahmani $R$, Just PA, Perret $C$. The $W n t / \beta$-catenin pathway as a therapeutic target in human hepatocellular carcinoma. Clin Res Hepatol Gastroenterol 2011;35:709-713.

30. Kurosu H, Yamamoto M, Clark JD et al. Suppression of aging in mice by the hormone Klotho. Science 2005;309:1829-1833.

31. Neidert MC, Sze L, Zwimpfer C et al. Soluble a-klotho: a novel serum biomarker for the activity of $\mathrm{GH}$-producing pituitary adenomas. Eur J Endocrinol 2013;168:575-583.

32. Yamazaki Y, Imura A, Urakawa I et al. Establishment of sandwich ELISA for soluble a-Klotho measurement: age-dependent change of soluble a-Klotho levels in healthy subjects. Biochem and Biophys Res Commun 2010;398:513-518.

33. Ohata $\mathrm{Y}$, Arahori $\mathrm{H}$, Namba $\mathrm{N}$ et al. Circulating levels of soluble a-Klotho are markedly elevated in human umbilical cord blood. J Clin Endocrinol Metab 2011;96:E943-E947.

34. Sze L, Bernays RL, Zwimpfer C et al. Excessively high soluble Klotho in patients with acromegaly. J Intern Med 2012;272:93-97.

35. Hernandez-Gea V, Toffanin S, Friedman SL et al. Role of the microenvironment in the pathogenesis and treatment of hepatocellular carcinoma. Gastroenterology 2013;144:512-527. 